\title{
CHARACTERISATION OF THE DIFFERENT STEPS DURING ZINC CHELATION INDUCED APOPTOSIS IN JURKAT AND HELA CELLS
}

\author{
F. Chimienti ${ }^{1}$, M. Sève ${ }^{1}$, S. Richard ${ }^{2}$, J. Mathieu $^{2}$, and A. Favier ${ }^{1}$ \\ ${ }^{1}$ Laboratoire de Biologie du Stress Oxydant (LBSO) \\ UJF Domaine de la Merci \\ 38700 La Tronche \\ ${ }^{2}$ Unité de Radiobiologie et inflammation \\ CRSSA, Avenue des Maquis du Grésivaudan \\ 38700 La Tronche
}

Zinc is an important component of the cell survival. It participates in several proteins structure and its intracellular concentration seems to be precisely regulated. Zinc is involved in apoptosis regulation and has been responsible for DNA fragmentation during apoptosis.

We try to define the mechanism of apoptosis induced by zinc deprivation and to determine the kinetic of the different steps in this type of apoptosis: morphological changes, nuceus condensation, DNA fragmentation, protein activation,....

Jurkat and HeLa cells were treated by the intracellular zinc chelator TPEN (N, N, $\mathrm{N}^{\prime}, \mathrm{N}^{\prime}$ tetrakis-(2 pyridylmethyl) ethylene diamine). The cells were then observed by phase-contrast and fluorescence microscopy, and harvested after 3, 6, 9, 12 or 24 hours of treatment.

We show that deprivation of zinc induces apoptosis in Jurkat and HeLa cells with a typical morphology. Zinc deprivation by TPEN treatment was monitored in HeLa cells, using a new Zn-specific fluorescent probe: Zinquin-ethyl-ester. Observation by phase contrast microscopy provide evidence that membrane blebbing occurs after 3 hours of TPEN treatment $(30 \mu \mathrm{M})$. Studies by fluorescence microscopy were assessed with DNA staining (Hoechst 33342). Nuclear condensation is visible after 6 hours of TPEN treatment andagarose gel electrophoresis confirms that DNA is fragmented after 6 hours.

Additionally, the caspase- 3 activity shows a huge increase with a maximum at 12 hours. By western-blot, we reveal the specific degradation of several transcription factors implicated in cell proliferation and differentiation.

We conclude that zinc seems to be involved in the regulation of apoptosis not only in endonuclease protection, but in upstream specific activation pathways. 